\title{
Treatment and Survival in Advanced Non-Small Cell Lung Cancer, Urothelial, Ovarian, Gastric and Kidney Cancer: A Nationwide Comprehensive Evaluation
}

\author{
Signe Sørup (D) \\ Bianka Darvalics (D) \\ Azza Ahmed Khalil (D) ${ }^{2}$ \\ Marianne Nordsmark ${ }^{2}$ \\ Mette $\mathrm{Hæe}^{2}$ \\ Frede Donskov ${ }^{3}$ \\ Mads Agerbæk (D) ${ }^{2}$ \\ Leo Russo ${ }^{4}$ \\ Dina Oksen (iD ${ }^{5}$ \\ Emmanuelle Boutmy (D) ${ }^{5}$ \\ Patrice Verpillat $\mathbb{D}^{5}$ \\ Deirdre Cronin-Fenton (ID) \\ 'Department of Clinical Epidemiology, \\ Department of Clinical Medicine, Aarhus \\ University \& Aarhus University Hospital, \\ Aarhus, Denmark; ${ }^{2}$ Department of \\ Oncology, Aarhus University Hospital, \\ Aarhus, Denmark; ${ }^{3}$ Department of \\ Clinical Medicine, Aarhus University, \\ Aarhus, Denmark; ${ }^{4}$ Worldwide Medical \\ and Safety, Pfizer, Collegeville, PA, USA; \\ ${ }^{5}$ Global Epidemiology, Merck Healthcare \\ $\mathrm{KGaA}$, Darmstadt, Germany
}

Purpose: Few studies have described real-world treatment patterns and survival before the widespread use of immune checkpoint inhibitors (ICIs). We aimed to describe anti-cancer treatment including the use of programmed cell death-1 and ligand-1 (PD-1/PD-L1) ICIs and overall survival (OS) in advanced cancer patients as a benchmarking real-world standard before widespread use of ICIs.

Patients and Methods: Using nationwide Danish medical registries, we assembled cohorts of Danish patients with advanced non-small cell lung cancer (NSCLC) $(n=12,283)$, urothelial carcinoma $(n=2504)$, epithelial ovarian cancer $(n=1466)$, gastric adenocarcinoma $(n=1457)$, and renal cell carcinoma (RCC) $(n=1261)$ diagnosed between 1/1/2013 and 31/12/2017. We describe anti-cancer treatment and OS using proportions, medians, and Kaplan-Meier methods. Results: Between 9\% (ovarian cancer) and 25\% (gastric adenocarcinoma) of patients did not receive anti-cancer treatment. The remaining patients received surgery, radiation therapy, and/or medical therapy. Chemotherapy was the most frequent medical therapy in all cohorts except for RCC (tyrosine kinase inhibitors). PD-L1/PD-1 ICIs were used in 7-8\% of the NSCLC and RCC cohorts - mainly as second or higher line treatments. OS was longest in patients starting treatment with surgery (eg 25.6 months [95\%-confidence interval (CI) $=21.9-29.4]$ for NSCLC and 21.4 months [95\%-CI=19.8-23.5] for urothelial carcinoma) and shortest for radiation therapy (eg 3.9 months [95\%-CI=3.6-4.2] for NSCLC and 12.6 months $[95 \%-\mathrm{CI}=9.2-17.5]$ for urothelial carcinoma). NSCLC patients starting with medical therapy had OS between these limits. Median OS for NSCLC patients starting treatment with PD-L1/PD-1 ICIs was 21.4 months (95\%-CI=13.9-not estimable).

Conclusion: Most patients with advanced NSCLC, urothelial carcinoma, epithelial ovarian cancer, gastric adenocarcinoma and $\mathrm{RCC}$ had poor OS in an era where only a minority received PD-L1/PD-1 ICIs. This information on treatment patterns and survival is important as a benchmarking real-world standard before widespread use of ICIs.

Keywords: cancer, treatment, survival, immunotherapy, programmed cell death-1 immune checkpoint inhibitors, programmed cell death-ligand-1 immune checkpoint inhibitors

\section{Introduction}

Surgery, radiation therapy, chemotherapy, and targeted therapy are traditional treatment modalities for cancer. ${ }^{1,2}$ Immunotherapy is increasingly used as a primary treatment and in treatment-refractory advanced disease. ${ }^{3}$ The immune checkpoint inhibitors (ICIs) targeting programmed cell death 1 (PD-1) and programmed cell death ligand 1 (PD- 
L1) block tumor-related downregulation of the immune system, thereby enhancing anti-tumor immunity. ${ }^{4}$ The first PD-L1 /PD-1 ICI was approved by the US Food and Drug administration in 2014 and by the European Medicines Agency in 2015 for advanced melanoma. ${ }^{3,5}$ Since then, these drugs have been approved for several cancers, including advanced nonsmall cell lung cancer (NSCLC), urothelial carcinoma, renal cell carcinoma (RCC), and gastric cancer, where PD-L1/PD-1 ICIs demonstrate prolonged survival. ${ }^{3,6-8}$ PD-L1/PD-1 ICIs are now standard care in Denmark for subgroups of patients with NSCLC, urothelial carcinoma, and RCC (Supplementary Table S1). PD-L1/PD-1 ICIs are being evaluated as treatments for advanced ovarian cancer. ${ }^{9}$

Given this changing treatment landscape, it is important to document the use of anti-cancer treatments in routine clinical care and overall survival across diverse cancers. This can function as a benchmark standard for evaluating the future treatment patterns and survival after the more widespread adoption of PD-L1/PD-1 ICIs into routine clinical care. This is particularly important because patients and treatment delivery often differ between routine clinical care and clinical trials. ${ }^{10}$ Therefore, we aimed to use Danish nationwide health registries to describe and graphically illustrate the real-world utilization and patterns of anti-cancer treatment, including the use of PD-L1/PD-1 ICIs and overall survival among patients with advanced NSCLC, urothelial, ovarian, gastric, and renal cell cancer.

\section{Materials and Methods}

The Danish national health care system provides the entire Danish population with unrestricted access to taxsupported health care and free-of-charge treatment at hospitals. ${ }^{11}$ The Danish Cancer Registry has collected information since $1943^{12}$ and the Danish National Patient Registry (DNPR) since 1977. ${ }^{13}$ All Danish residents are assigned a unique personal identification number, which can be used to link information in these registers and the Danish Civil Registration System, which records information on immigrations and deaths for Danish residents. ${ }^{14,15}$ We utilized these resources to perform this nationwide, register-based study including 5 epidemiological cohorts of cancer patients in Denmark as outlined below.

\section{Population and Follow-Up}

We selected five cohorts of patients with advanced cancer diagnosed between January 1, 2013 and December 31, 2017 based on data from the Danish Cancer Registry on International Classification of Disease (ICD) version 10
(ICD-10) codes, ICD-Oncology version 3 (ICD-O3) morphology codes, and stage of cancer defined according to the 7th Edition of the American Joint Committee on Cancer (AJCC) Cancer Staging Manual using variables on tumor $(\mathrm{T})$, node $(\mathrm{N})$, and metastasis $(\mathrm{M}) .^{12,16}$

We included NSCLC, epithelial ovarian cancer, gastric adenocarcinoma and RCC patients with AJCC stage III and IV at the time of diagnosis. For urothelial carcinoma, we included AJCC stage II, III, and IV (muscle-invasive and metastatic urothelial carcinoma) because the European Association of Urology divide their treatment guidelines for bladder cancer into "Non-muscle-invasive bladder cancer" and "Muscle-invasive and metastatic bladder cancer". ${ }^{17}$ Where information was missing on one or more of the TNM variables, we included patients if the available TNM information was sufficient to indicate that the patient had at least AJCC stage III (stage II for urothelial carcinoma). The specific criteria for each cohort are outlined in Figure 1. The Danish Cancer Registry only registers information about the primary tumor at diagnosis; therefore, we could not include patients who progressed to advanced disease after diagnosis.

We followed all cohorts of patients from the date of diagnosis to death, emigration, new primary cancer, or last date with available data (December 31, 2018). We censored on new primary cancers, as this may affect subsequent treatment patterns and survival.

\section{Patient Characteristics}

We included gender, age, AJCC stage, year of cancer diagnosis, and Charlson co-morbidity index at the time of diagnosis. The Charlson co-morbidity index is a weighted index of comorbidities, which we calculated using ICD-8 and ICD-10 diagnoses in the DNPR from 1977 until and including the date of inclusion in this study, excluding cancer diagnoses. ${ }^{18}$

We obtained information about tests for PD-L1 mutations from the Danish National Pathology Registry. ${ }^{19,20}$

\section{Anti-Cancer Treatment and Procedures}

We retrieved information on anti-cancer treatments and procedures from the DNPR from the date of diagnosis until end of follow-up. We included therapeutic intent surgeries for each cancer and we defined radiation therapy and medical therapy (overall and subdivided into different types) using treatment codes from the DNPR (Supplementary Coding Appendix). The treatment codes included specific (eg 


\begin{tabular}{|c|c|c|c|c|c|}
\hline $\begin{array}{l}\text { Lung cancer } \\
\text { diagnosed 2013-2017 } \\
\text { according to the DCR } \\
\text { (ICD-10 code: C34) } \\
\text { and included in the } \\
\text { DCRS at the time of } \\
\text { diagnosis } \\
\mathbf{n = 2 3 , 7 1 0}\end{array}$ & $\begin{array}{c}\text { Bladder and } \\
\text { urothelial cancer } \\
\text { diagnosed } 2013-2017 \\
\text { according to the DCR } \\
\text { (ICD-10 codes: C65- } \\
\text { C68.0) and included in } \\
\text { the DCRS at the time } \\
\text { of diagnosis } \\
\mathbf{n}=5369\end{array}$ & $\begin{array}{c}\text { Ovarian cancer } \\
\text { diagnosed 2013-2017 } \\
\text { according to the DCR } \\
\text { (ICD-10 code: C56) } \\
\text { and included in the } \\
\text { DCRS at the time of } \\
\text { diagnosis } \\
\mathbf{n = 2 4 6 8}\end{array}$ & $\begin{array}{c}\text { Gastric cancer } \\
\text { diagnosed 2013-2017 } \\
\text { according to the DCR } \\
\text { (ICD-10 code: C16) } \\
\text { and included in the } \\
\text { DCRS at the time of } \\
\text { diagnosis } \\
\mathbf{n = 3 0 8 6}\end{array}$ & $\begin{array}{l}\text { Renal cancer } \\
\text { diagnosed 2013-2017 } \\
\text { according to the DCR } \\
\text { (ICD10 code: C64) and } \\
\text { included in the DCRS } \\
\text { at the time of diagnosis } \\
\text { n= } \mathbf{4 7 1 4}\end{array}$ & $\begin{array}{r}39,347 \text { patients } \\
\text { identified in DCR }\end{array}$ \\
\hline $\begin{array}{c}\text { Not AJCC stage III } \\
\text { or IV at the time of } \\
\text { diagnosis } \\
n=6963\end{array}$ & $\begin{array}{c}\text { Not AJCC stage II, } \\
\text { III or IV at the time } \\
\text { of diagnosis } \\
\mathbf{n = 2 4 8 8}\end{array}$ & $\begin{array}{c}\text { Not AJCC stage III } \\
\text { or IV at the time of } \\
\text { diagnosis } \\
\mathbf{n = 8 9 0}\end{array}$ & $\begin{array}{c}\text { Not AJCC stage III } \\
\text { or IV at the time of } \\
\text { diagnosis } \\
n=1462\end{array}$ & $\begin{array}{c}\text { Not AJCC stage III } \\
\text { or IV at the time of } \\
\text { diagnosis } \\
n=3154\end{array}$ & $\begin{array}{l}14,957 \text { patients } \\
\text { excluded based } \\
\text { on AJCC stage }\end{array}$ \\
\hline $\begin{array}{c}\text { AJCC stage III or IV at } \\
\text { the time of diagnosis } \\
n=16,747\end{array}$ & $\begin{array}{c}\text { AJCC stage II, III or IV } \\
\text { at the time of diagnosis } \\
n=\mathbf{2 8 8 1}\end{array}$ & $\begin{array}{l}\text { AJCC stage III or IV at } \\
\text { the time of diagnosis } \\
n=1578\end{array}$ & $\begin{array}{c}\text { AJCC stage III or IV at } \\
\text { the time of diagnosis } \\
n=1624\end{array}$ & $\begin{array}{c}\text { AJCC stage III or IV at } \\
\text { the time of diagnosis } \\
n=1560\end{array}$ & $\begin{array}{r}24,390 \text { patients } \\
\text { with the included } \\
\text { AJCC cancer stages }\end{array}$ \\
\hline $\begin{array}{l}\text { Not morphology } \\
\text { codes }^{\mathrm{a}} \text { for non- } \\
\text { small cell lung } \\
\text { cancer } \\
\mathbf{n = 4 4 6 4}\end{array}$ & $\begin{array}{l}\text { Not morphology } \\
\text { codes }^{\mathrm{b}} \text { for first } \\
\text { time } e^{\mathrm{c}} \text { transitional } \\
\text { cell carcinoma } \\
\mathbf{n}=\mathbf{3 7 7}\end{array}$ & $\begin{array}{l}\text { Not morphology } \\
\text { codes }^{\mathrm{d}} \text { for } \\
\text { epithelial cancer } \\
\text { n=112 }\end{array}$ & $\begin{array}{l}\text { Not morphology } \\
\text { codes }^{\text {for }} \\
\text { adenocarcinoma } \\
\text { n=167 }\end{array}$ & $\begin{array}{l}\text { Not morphology } \\
\text { codes }^{f} \text { for clear } \\
\text { cell carcinoma } \\
\text { n=299 }\end{array}$ & $\begin{array}{r}5419 \text { patients } \\
\text { excluded based } \\
\text { morphology code }\end{array}$ \\
\hline $\begin{array}{l}\text { Included patients with } \\
\text { non-small cell lung } \\
\text { cancer AJCC stage III } \\
\text { and IV at the time of } \\
\text { diagnosis } \\
n=12,283\end{array}$ & $\begin{array}{c}\text { Included patients with } \\
\text { urothelial carcinoma } \\
\text { AJCC stage II, III and } \\
\text { IV at the time of } \\
\text { diagnosis } \\
\mathbf{n = 2 5 0 4}\end{array}$ & $\begin{array}{l}\text { Included patients with } \\
\text { epithelial ovarian } \\
\text { cancer AJCC stage III } \\
\text { and IV at the time of } \\
\text { diagnosis } \\
n=1466\end{array}$ & $\begin{array}{c}\text { Included patients with } \\
\text { gastric } \\
\text { adenocarcinoma } \\
\text { AJCC stage III and IV } \\
\text { at the time of diagnosis } \\
n=1457\end{array}$ & $\begin{array}{c}\text { Included patients with } \\
\text { renal cell carcinoma } \\
\text { AJCC stage III and IV } \\
\text { at the time of diagnosis } \\
n=1261\end{array}$ & $\begin{array}{l}18,971 \text { patients } \\
\text { included in the study }\end{array}$ \\
\hline
\end{tabular}

Figure I Flowchart of selection of the patients with advanced non-small cell lung cancer, urothelial carcinoma, epithelial ovarian cancer, gastric adenocarcinoma, or renal cell carcinoma included in the study.

Notes: Stage was defined according to the 7th Edition of the AJCC Cancer Staging Manual. a) ICD-O3 morphology codes different from: 8I,403, 80,703, 80,463, 80, I33, $82,303,85,603,80,833,84,803,82,603,80,333,80,123,80,713,82,503,80,723,81,233,80,743,80,323,80,143,80,763$, 80,I03, 80,203. b) ICD-O3 morphology codes different from: $81,203,81,223,81,313$. c) Sixteen patients had two registrations with bladder or urothelial transitional cell carcinoma AJCC stage II, III, and IV in DCR, we excluded the latest of these registrations. d) ICD-O3 morphology codes different from: 80,103, 80,203, 80,703, 81,203, 8I,403, 82,303, 82,403, 82,463, 82,493, 82,603, $83,103,83,803,84,413,84,503,84,603,84,613,84,703,84,743,84,803,84,903,85,603$. e) ICD-O3 morphology codes different from: 8I,403, 81,433, 8I,443, 8I,453, 82, I03, 82,II3, 82,603, 83, I03, 84,803, 84,903, 82,303. f) ICD-O3 morphology codes different from: 83, I03, 83,I23.

Abbreviations: AJCC, American Joint Committee on Cancer; DCR, Danish Cancer Registry; DCRS, Danish Civil Registration System; ICD-I0, international classification of diseases tenth revision; ICD-O3, international classification of diseases-Oncology version 3; n, number of patients.

"treatment with cisplatin") and non-specific codes (eg “cytostatic treatment"). In addition to groups of specific medical therapy types (eg chemotherapy and tyrosine kinase inhibitors), we included "unspecified medical therapy" consisting of non-specific codes and "other medical therapy" consisting of treatment codes indicating specific drugs or drug classes, which are uncommon treatments for the included cancers. We identified lines of medical therapy in the DNPR using a validated algorithm, which incorporated information on chemotherapy and targeted therapies (manuscript in preparation). We revised the algorithm to also include all the medical therapies included in this study (revised algorithm published on GiTHub: https:/github.com/BiankaDarvalics/ medical therapy_line_algorithm/tree/1.0.0 [doi: 10.5281/ zenodo.5495900]).

We also outlined the number of patients who received at least one treatment with a PD-L1/PD-1 ICI (avelumab, atezolizumab, durvalumab, nivolumab, pembrolizumab).

\section{Ethics}

Under Danish law, ethical permission is not required for registry-based research. This study followed the EU General Data Protection Regulation. Data was obtained from the Danish Health Data Authority.

\section{Statistical Methods}

Analyses were performed separately for each cohort. First, we described the characteristics of the cohort, followed by the utilization of anti-cancer treatment and procedures and types of medical therapy using proportions, means, and medians as appropriate and illustrated graphically with horizontally stacked bar charts, density plots, and bar charts. We created Sankey plots to illustrate patient transition through different types of treatments. These plots included surgery, radiation therapy and the most frequent medical therapy for each cohort, including the remaining medical therapies in the 
"other medical therapy group". However, we included PDL1/PD-1 ICIs as a separate group for each cancer to show the extent of use as anti-cancer treatment. For the NSCLC cohort and urothelial carcinoma cohort, we also created Sankey plots separately for patients diagnosed in 2017 to show the use of PD-L1/PD-1 ICIs in more recently diagnosed patients.

We used Kaplan-Meier plots to illustrate overall survival since diagnosis with all-cause death from the Danish Civil Registration System as the event and censoring at the end of follow-up. ${ }^{21}$ We also used Kaplan-Meier plots of overall survival from the date of first anti-cancer treatment according to the main groups of the first anti-cancer treatment with enough patients to ensure anonymity. In supplemental analyses, we stratified these Kaplan-Meier plots by stage at diagnosis.

We used SAS version 9.4 for analyses and $\mathrm{R}$ version 4.0.2 for visualizations.

\section{Results}

We included 18,971 cancer patients (Figure 1) which are characterized in Figure 2 and Supplementary Table S2. Treatment patterns and survival are described below by cohort.

\section{NSCLC}

Among 12,283 patients with AJCC stage III and IV NSCLC, $20 \%$ did not receive anti-cancer treatment, while around $60 \%$ received radiation therapy and medical therapy (mainly chemotherapy) at least once (Figure 3 and 4, and Supplementary Table S3). Around 7\% got PD-L1 /PD-1 ICIs at least once with a median of 32 days from diagnosis to first treatment with PD-L1/PD-1 ICIs (quartile 1-quartile 3=23-48; Figure 4 and Supplementary Tables S3 and $\underline{\mathrm{S}}$ ). Chemotherapy was the most frequent first anticancer treatment; $2 \%$ received PD-L1/PD-1 ICIs as the first anti-cancer treatment (Supplementary Figure S1). When restricting to patients diagnosed in 2017, 8\% received PD-L1/PD-1 ICIs as the first treatment (Supplementary Figure S2). The median overall survival time from date of NSCLC diagnosis was 8.0 months (95\%-confidence interval $(\mathrm{CI})=7.8-8.3$ ) but was considerably longer for patients with stage III at diagnosis (17.0 months; 95\%-CI=16.2-17.9) compared with stage IV at diagnosis (5.7 months; 95\%-CI=5.4-5.9; Figure 5 and Supplementary Table S6). The median overall survival from date of initiation of the first anti-cancer treatment was longest for patients with surgery as the first treatment (25.6 months; 95\%-CI $=21.9-29.4$ ) followed by patients with PD-L1/PD-1 ICIs (21.4 months; 95\%-CI=13.9-not estimable) and shortest for patients who received only radiation therapy (3.9 months; $95 \%$-CI=3.6-4.2; Figure 5 and Supplementary Table S6). More patients with stage III received surgery as a first treatment compared with stage IV (Supplementary Table S5); for stage IV, the median survival was longer for patients receiving PD-L1/PD-1 ICIs and tyrosine kinase inhibitors than for patients

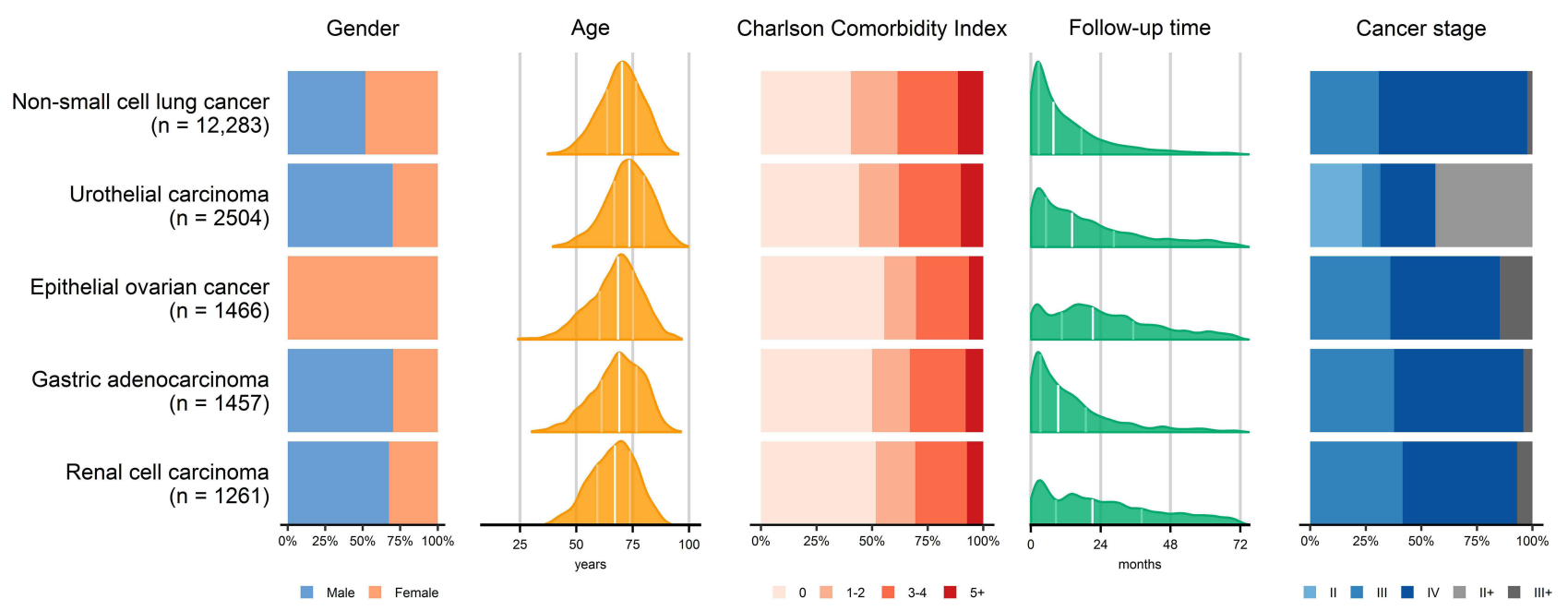

Figure 2 Patient characteristics.

Notes: In the plots for age and follow-up time, the first quartile, median, and third quartile are indicated by vertical lines. Stage is defined according to the 7th Edition of the AJCC Cancer Staging Manual based on information on tumor size (T), node status (N), and metastasis (M). However, information on one or more of the TNM variables could be missing. If the available information indicated that the patient was at least stage II, it is indicated by "II+" and similar definition for "III+". Exact numbers, proportions, and medians can be found in Supplementary Table S2 along with information on the year of diagnosis, and test for PD-LI mutations.

Abbreviations: AJCC, American Joint Committee on Cancer; $n$, number of patients. 


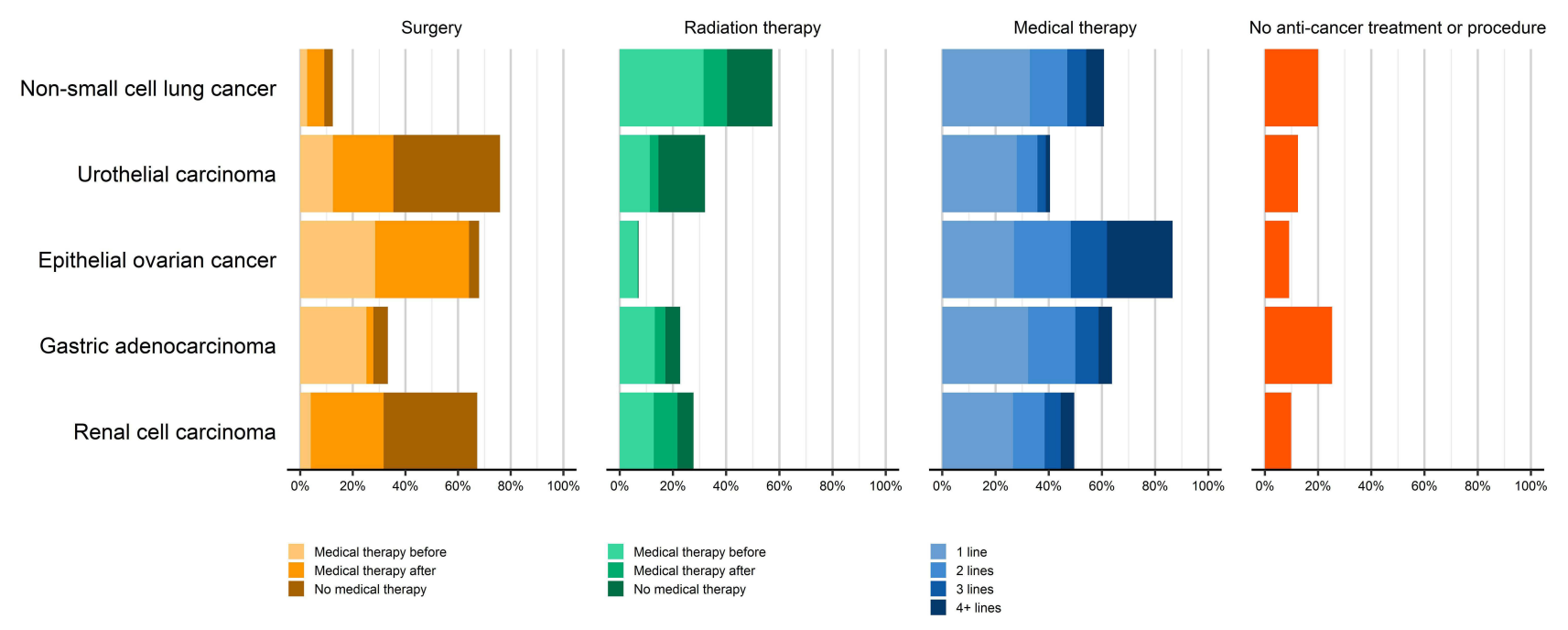

Figure 3 Anti-cancer treatment and procedures.

Notes: "Medical therapy before" indicates first administration of medical therapy before the first surgery or radiation therapy, respectively. "Medical therapy after" indicates first administration of medical therapy after the first surgery or radiation therapy, respectively. "Medical therapy" include chemotherapy, PARP inhibitors, HER-2 antibody, angiogenesis inhibitors, interferon-alfa/interleukin-2, PD-LI/PD-I ICI, BCG, TKI, other targeted therapies, treatment with other antibodies, treatment with other immunomodulatory agents, and treatment with other biologically modified substances.

Abbreviations: BCG, Bacille Calmette Guerin; HER-2, human epidermal growth factor receptor 2; ICl, immune checkpoint inhibitor; PARP, poly ADP ribose polymerase; PD-I, Programmed cell death receptor I; PD-LI, Programmed cell death receptor ligand I; TKI, tyrosine kinase inhibitor.

initiating treatment with surgery (Figure 6 and Supplementary Table S6).

\section{Urothelial Carcinoma}

Among the 2504 patients with AJCC stage II, III, and IV urothelial carcinoma, $13 \%$ did not undergo anti-cancer treatment (Figure 3). Around $76 \%$ of the patients received surgery, over half of whom did not receive any medical therapy either before or after the surgery (Figure 3 and Supplementary Table S3). Overall, 40\% received medical therapy at least once, most often chemotherapy; $3 \%$ received PD-L1/PD-1 ICIs at least once (Figure 3, Figure 4, and Supplementary Table S3). Among patients initiating anti-cancer treatments, $75 \%$ started with surgery (Supplementary Figure S3). When restricting to patients diagnosed in 2017, approximately 3\% received PD-L1 /PD-1 ICIs as the second treatment (Supplementary Figure S4). The median overall survival time from the date of cancer diagnosis was 19.6 months (95\%-CI =17.9-20.9), but considerably shorter for patients with stage IV at diagnosis (9.4 months; 95\%-CI=8.3-10.6) compared with stage III patients (29.9 months; 95\%-CI =22.5-40.5; Figure 5 and Supplementary Table S6). The median overall survival from the date of initiation of the first anti-cancer treatment was similar for patients starting with surgery (21.4 months; 95\%-CI=19.8-23.5) or starting with chemotherapy (20.6 months; 95\%-CI=15.4-23.3) but shorter for patients starting with radiation therapy (12.6 months, 95\%-CI=9.2-17.5; Figure 5 and Supplementary Table S6). However, stage III patients starting with chemotherapy had a longer median survival than patients starting with surgery or radiation therapy (Figure 6 and Supplementary Table S6).

\section{Epithelial Ovarian Cancer}

Of the 1466 patients with AJCC stage III and IV epithelial ovarian cancer, $9 \%$ did not receive anti-cancer treatments, $68 \%$ underwent surgery, and $87 \%$ received medical therapy at least once with $28 \%$ receiving at least four lines (Figure 3 and Supplementary Table S3). Most patients received chemotherapy, 25\% received angiogenesis inhibitors, and less than $1 \%$ received PD-L1/PD-1 ICIs (Figure 4). About half of the patients had chemotherapy as the first anti-cancer treatment, while the other half underwent surgery, often followed by chemotherapy (Supplementary Figure S5). The median overall survival time from the date of cancer diagnosis was 40.2 months (95\%-CI=34.0-44.6) for stage III and 22.9 months $(95 \%$ $\mathrm{CI}=20.6-26.4$ ) for stage IV (Figure 5 and Supplementary Table S6). The median overall survival from the date of initiation of the first anti-cancer treatment was longer for patients starting with surgery (43.5 months; 95\%-CI $=39.4$-not estimable) than for patients starting with 

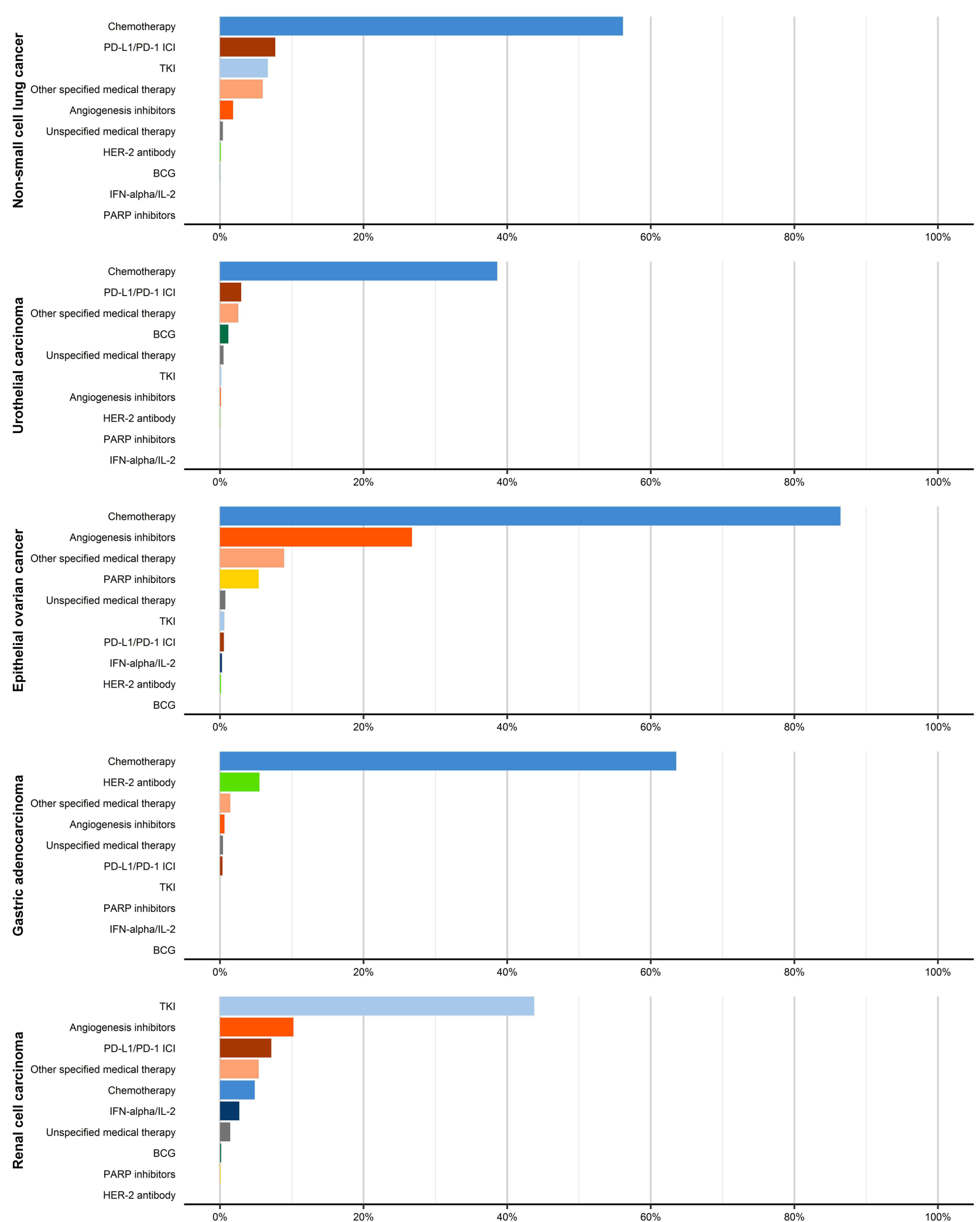

Figure 4 Proportion of patients receiving the given medical therapy group at least once during follow-up.

Notes: "Other specified medical therapy" include registrations with codes indicating specific drug types that are not included in any of the main groups eg other monoclonal antibodies or other interferons. "unspecified medical therapy" include registrations with codes not indicating any specific drug types eg "cytostatic treatment", "biologically modified substances" etc.

Abbreviations: BCG, Bacille Calmette Guerin; HER-2, human epidermal growth factor receptor 2; ICI, immune checkpoint inhibitor; IFN, interferon; IL, interleukin; PARP, poly ADP ribose polymerase; PD-I, Programmed cell death receptor I; PD-LI, Programmed cell death receptor ligand I; TKI, tyrosine kinase inhibitor. 
From date of diagnosis
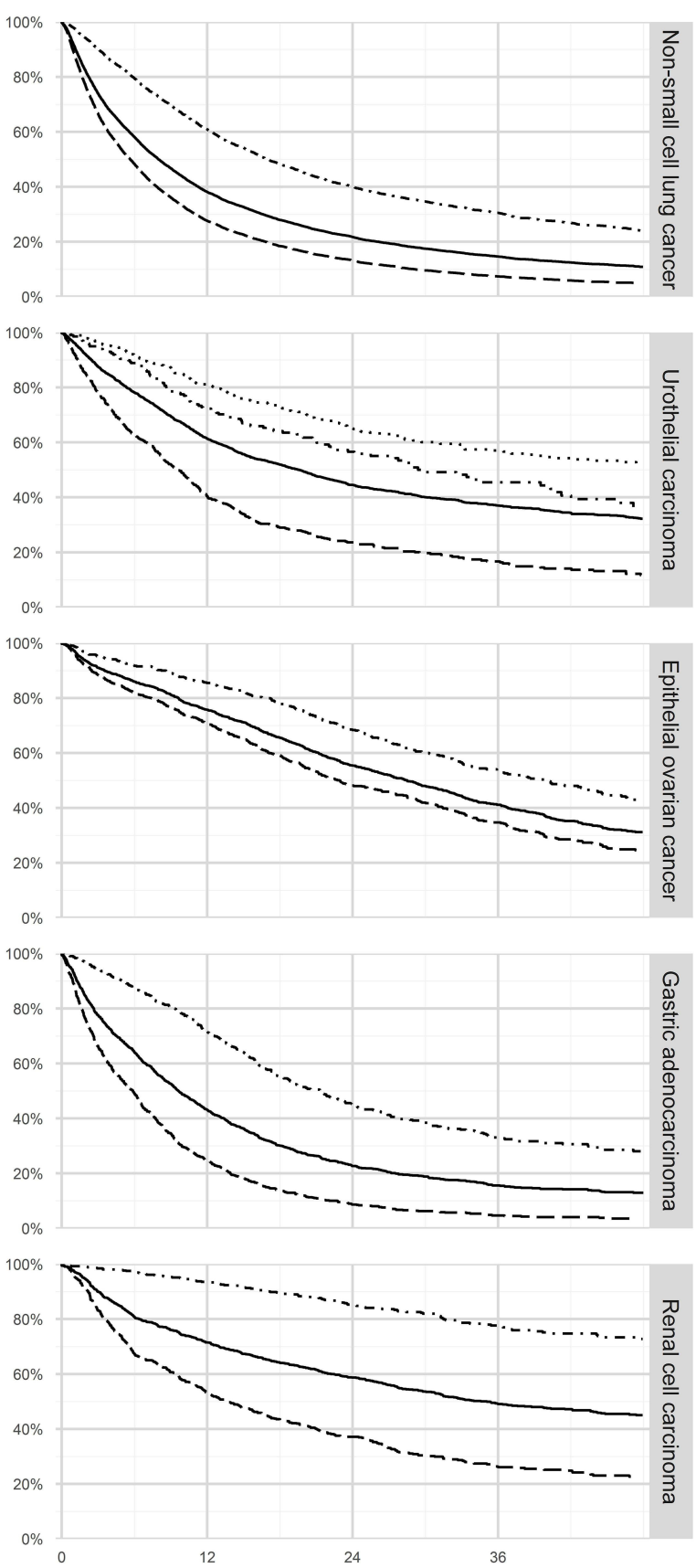

- All $\cdots$ II $\cdot-\cdot$ III -- IV
From date of first anti-cancer treatment

or procedure
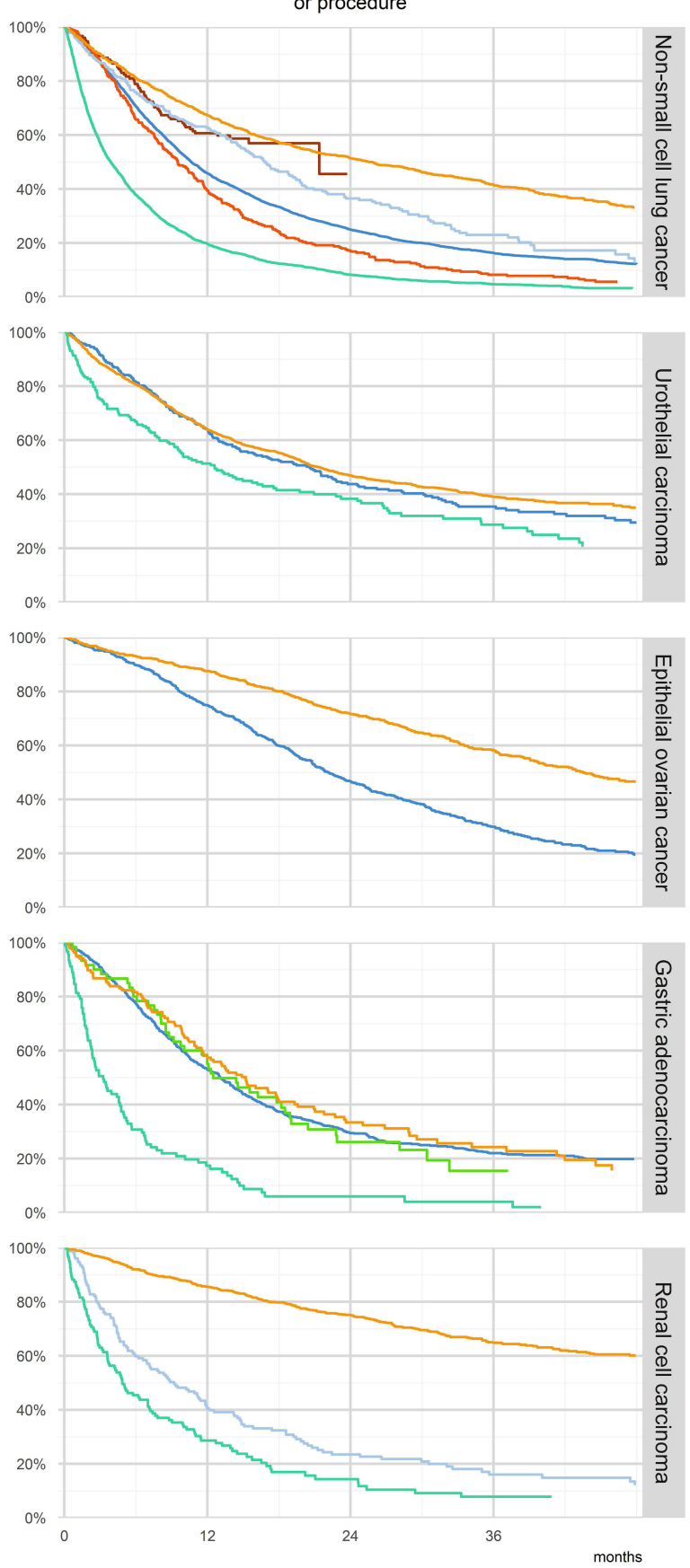

$$
\begin{array}{lllll}
- \text { PD-L1/PD-1 } & - \text { Chemo+Al } & & & \text { TKI }- \text { RT } \\
\text { Chemo } & - & \text { Chemo+HER-2 } & - & \text { Surgery }
\end{array}
$$

Figure 5 Kaplan-Meier plots of overall survival from date of diagnosis overall and according to AJCC stage and from date of initiation of first anti-cancer treatment or procedure according to type of first anti-cancer treatment or procedure.

Notes: Kaplan-Meier curves treating dates of migration, new primary cancer, and end of follow-up as censoring events. The heading indicates from which date follow-up starts in each plot. The plot "From date of first anti-cancer treatment or procedure" includes only the most frequent types of first anti-cancer treatment or procedure for each type of cancer to ensure there is no violation of privacy. Supplementary Table S4 gives the number of days between diagnosis and initiation of the first anti-cancer treatment, Supplementary Table S5 gives the number of persons and events for the plots, and Supplementary Table S6 gives the median survival.

Abbreviations: Al, angiogenesis inhibitor; AJCC, American Joint Committee on Cancer; Chemo, chemotherapy; HER-2, human epidermal growth factor receptor 2 antibody; PD-LI /PD-I, Programmed cell death receptor ligand I/Programmed cell death receptor I immune checkpoint inhibitor; RT, radiation therapy; TKI, tyrosine kinase inhibitor. 
chemotherapy (22.2 months; 95\%-CI=20.8-24.5; Figure 5 and Supplementary Table S6).

\section{Gastric Adenocarcinoma}

Of the 1457 patients with AJCC stage III and IV gastric adenocarcinoma, $25 \%$ did not receive anti-cancer treatment, $64 \%$ received medical therapy (mainly chemotherapy) at least once, and less than 1\% received PD-L1/PD-1 ICIs (Figure 3 and 4). Chemotherapy was part of the first anticancer treatment given to $75 \%$ of the patients who initiated treatment (Supplementary Figure S6). The median overall survival time from the date of cancer diagnosis was 21.4 months $(95 \%-\mathrm{CI}=18.6-23.4)$ for stage III and 5.9 months (95\%-CI $=5.1-6.3$ ) for stage IV (Figure 5 and Supplementary Table S6). The median overall survival from date of initiation of first anti-cancer treatment was longest for patients starting with surgery (15.2 months; 95\%-CI=11.7-18.0), but when stratifying by stage the median overall survival was longest for chemotherapy in stage III (25.4 months; 95\%-CI=21.331.3) and longest for chemotherapy+HER-2 in stage IV disease (12.0 months; 95\%-CI=8.5-16.2; Figure 5, Figure 6, and Supplementary Table S6).

\section{$\mathrm{RCC}$}

Of the 1261 patients with AJCC stage III and IV RCC, $10 \%$ did not receive anti-cancer treatment, $67 \%$ received surgery and $50 \%$ received medical therapy at least once mainly tyrosine kinase inhibitors (Figure 3, Figure 4, Supplementary Table S3). Among the patients initiating anti-cancer treatments, $73 \%$ started with surgery (Supplementary Figure S7). PD-L1/PD-1 ICIs were given to $7 \%$ of the patients, mainly after initial treatment with surgery or other medical therapies (Figure 4, Supplementary Table S3, and Supplementary Figure S7). The median overall survival time from the date of cancer diagnosis was 35.1 months $(95 \%-\mathrm{CI}=30.8-42.2)$, but shorter for patients with stage IV (13.6 months; 95\%-CI $=11.8-16.2$ ) compared with stage III disease (Figure 5 and Supplementary Table S6). The overall survival from the date of initiation of the first anti-cancer treatment was the longest for patients starting with surgery, where median survival was not reached during follow-up (Figure 5). Compared with this, the median survival was markedly shorter for patients starting with radiation therapy as first treatment (4.9 months; 95\%-CI=3.6-7.0) and tyrosine kinase inhibitors (9.2 months; $95 \%-\mathrm{CI}=6.9-11.8$; Figure 5 and Supplementary Table S6).

\section{Discussion}

Across our panel of diverse advanced cancer cohorts, a considerable proportion of patients did not receive any anti-cancer therapy. The remaining patients received surgery, radiation therapy, and/or medical therapy. The use of medical therapy was most intense in patients with epithelial ovarian cancer and lowest in urothelial carcinoma. Use of PD-L1/PD-1 ICIs was rare, but largest among patients with NSCLC or RCC. A minority of these patients received PD-L1/PD-1 ICIs, mainly subsequent to other anti-cancer treatments. However, among NSCLC patients diagnosed in 2017, the proportion with PD-L1/PD-1 ICIs as first treatment increased. Patients undergoing surgery as the first anti-cancer treatment or procedure had the longest overall survival; those starting with radiation therapy had the shortest overall survival. Patients starting with medical therapy had overall survival between these limits varying by cohort and type of first medical therapy. In the NSCLC cohort, patients starting with PD-L1/PD-1 ICIs had the second longest median overall survival. However, when stratifying the survival curves by stage at diagnosis, the median survival was longer after medical therapy than after surgery in a few instances.

\section{Strengths and Weaknesses}

Several issues should be considered when interpreting our findings. We used nationwide registries, representing realworld clinical practice in Denmark with virtually complete coverage and follow-up. ${ }^{15}$ This study only included patients with advanced cancer at the time of diagnosis. We did not know if patients were treated with curative or palliative intent and information on cancer progression was not available. Patients who progress to an advanced stage after diagnosis may have different characteristics, treatment patterns, and survival compared with patients initially diagnosed with advanced stage. There was no information on number of lines of medical therapy in the DNPR. We therefore modified a previously validated algorithm to capture the lines of medical therapy (Sorup et al [manuscript in preparation]). Several aspects of the included registers have been validated, ${ }^{11,13}$ we note a high positive predictive value of treatment codes for medical therapy ${ }^{22,23}$ in the DNPR and diagnosis codes and morphology codes in the Danish Cancer Registry. ${ }^{24}$ However, the completeness of TNM codes in the Danish Cancer Registry is lower for older patients and/or those with extensive comorbidity, ${ }^{25}$ which could lead to selection bias favoring younger and less co-morbid patients in the present study. 
Stage II
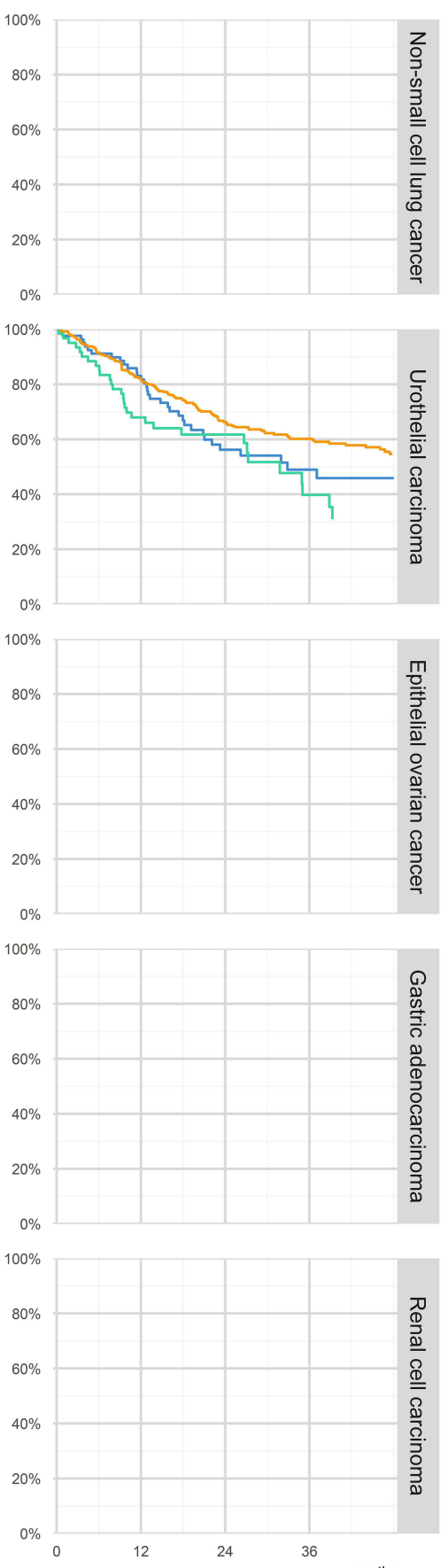

Stage III

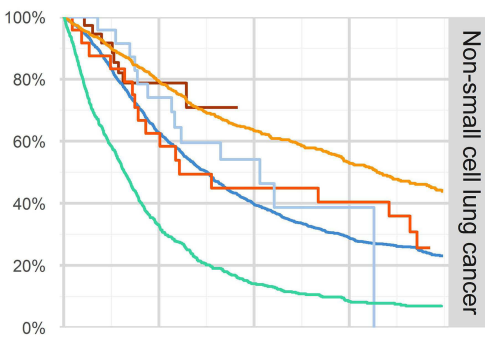

$100 \%$
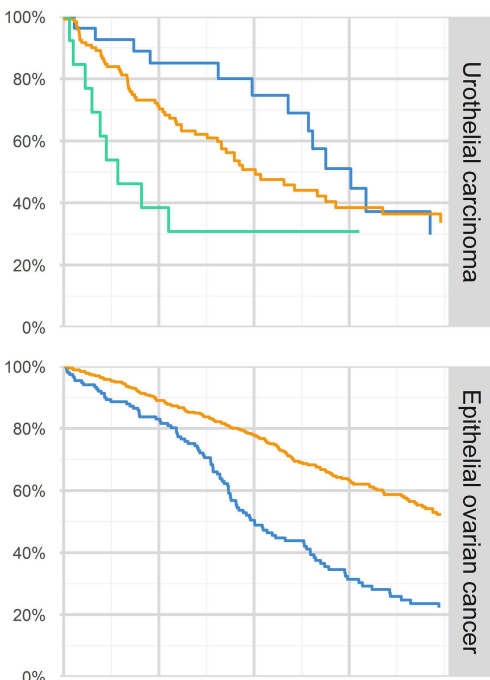

$100 \%$

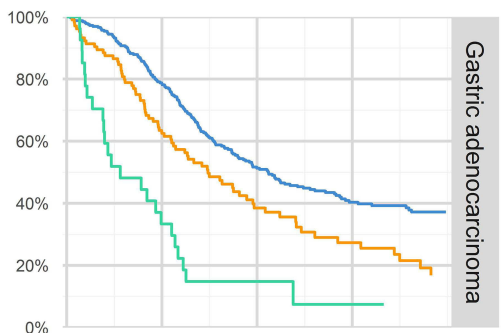

$100 \%$

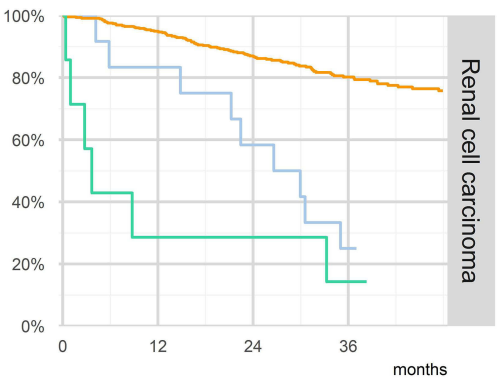

- PD-L1/PD-1 - Chemo+Al - TKI $\quad-$ RT
Stage IV
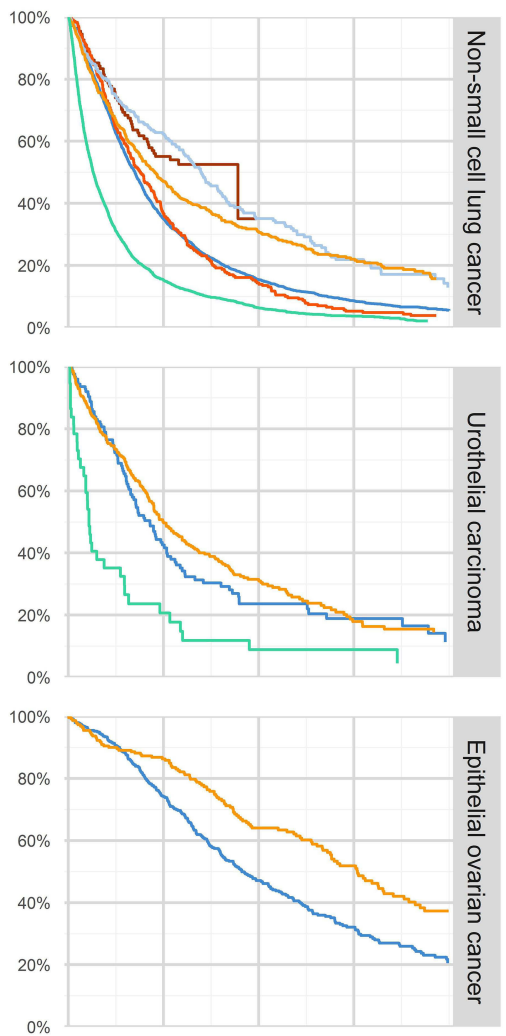
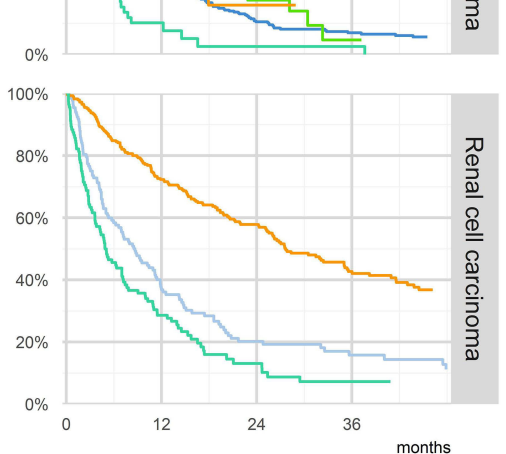

Figure 6 Kaplan-Meier plots of overall survival from date of initiation of first anti-cancer treatment or procedure according to type of first anti-cancer treatment or procedure stratified by AJCC stage.

Notes: Kaplan-Meier curves treating dates of migration, new primary cancer, and end of follow-up as censoring events. The plots include only the most frequent types of first anti-cancer treatment or procedure for each type of cancer to ensure there is no violation of privacy. Supplementary Table S5 gives the number of persons and events for the plots and Supplementary Table $\mathrm{S} 6$ gives the median survival.

Abbreviations: Al, angiogenesis inhibitor; AJCC, American Joint Committee on Cancer; Chemo, chemotherapy; HER-2, human epidermal growth factor receptor 2 antibody; PD-LI/PD-I, Programmed cell death receptor ligand I/Programmed cell death receptor I immune checkpoint inhibitor; RT, radiation therapy; TKI, tyrosine kinase inhibitor. 


\section{Comparison with Other Studies}

Overall, our observed main anti-cancer treatment patterns reflect those reported elsewhere. ${ }^{26-30}$ A notable exception is a US-based study of patients with stage IV NSCLC diagnosed 2013-2016, where PD-L1/PD-1 inhibitors were most frequent in second- and third-line medical therapy. ${ }^{31}$ This may reflect later approval and a more restrictive introduction of $\mathrm{PD}-\mathrm{L} 1 / \mathrm{PD}-1$ inhibitors in Denmark, where inclusion of new anti-cancer medical therapy in standard care has been based on national recommendations since $2012 .^{32,33}$ The stage IV urothelial cancer patients had similar median overall survival after the first chemotherapy as in a Dutch study. ${ }^{34}$

\section{Interpretation}

Overall, our findings highlight the chronic nature and poor prognosis of advanced cancer. Patients who received anticancer treatment transitioned through several treatment modalities, illustrating the limited effectiveness of current anti-cancer treatment. Furthermore, not all patients initiated anti-cancer treatment. We did not have any information about reasons for non-treatment, but it could be related to very advanced disease and/or significant comorbidities; a previous study found that non-treatment was related to patient preferences and contraindications against treatment. ${ }^{35}$ Immunotherapies, such as PD-L1 /PD-1 ICIs may prolong survival with reduced toxicity. ${ }^{3,36}$ Our study highlights the gradual uptake of PDL1/PD-1 ICIs into standard clinical care. PD-L1/PD-1 ICIs were first recommended in Denmark for a subgroup of NSCLC patients in 2015, followed by recommendations for subgroups of RCC patients in 2016 and urothelial carcinoma patients in 2017 (Supplementary Table S1). These explain the low uptake across our study cohorts. The NSCLC cohort included enough patients initiating treatment with PD-L1/PD-1 ICIs to estimate survival and showed one of the longest median survivals, although the interpretation was limited by the short follow-up. However, it is important to note that the differences in overall survival according to the first anti-cancer treatment cannot be interpreted as causal differences between different treatments and could depend on subsequent treatments. Instead, these are likely to reflect disease severity, comorbidities, and performance status of the patients, which determines treatment choice. For example, median survival was shorter in patients with radiation therapy as a first treatment, which may indicate its palliative intent. ${ }^{37}$

\section{Conclusion}

Most patients with advanced NSCLC, urothelial carcinoma, epithelial ovarian cancer, gastric adenocarcinoma and RCC received anti-cancer treatment; however, a considerable proportion of the patients did not receive any anti-cancer treatment. Chemotherapy was the most frequently used medical therapy in all cohorts except for RCC, where tyrosine kinase inhibitors were most frequent. PD-L1/PD-1 ICIs were most frequent in NSCLC and RCC cohorts and were mainly administered after other anti-cancer treatments. The overall survival was poor, but it varied by type of cancer, stage and first anti-cancer treatment. The survival estimates can be used as information on expected survival after diagnosis or initiation of different types of anti-cancer treatment in a real-world setting, under due consideration of differences related to setting, patient population and characteristics, and time period.

This study demonstrates how real-world data can be used to document and illustrate treatment patterns and survival in standard cancer care. The results can serve as an important benchmark for evaluating the effectiveness of introducing PD-L1/PD-1 ICIs and other novel treatments into standard cancer care.

\section{Data Sharing Statement}

The data was obtained from the Danish Health Data Authority. We are not allowed to share the data, but the same registry data can be obtained from the Danish Health Data Authority by other researchers fulfilling the requirements.

\section{Ethics Approval and Informed Consent}

Under Danish law, ethical permission is not required for registry-based research and it is not required to obtain consent from participants. This study followed the EU General Data Protection Regulation.

\section{Acknowledgments}

This work was partly supported by Merck (CrossRef Funder ID: $10.13039 / 100009945)$ by an institutional grant to and administered by Aarhus University Hospital, as part of an alliance between Merck KGaA, Darmstadt, Germany and Pfizer Inc., New York, NY, USA.

\section{Author Contributions}

SS and DCF conceived and designed the study, acquired data and interpreted the results. BD designed the study, acquired data and interpreted the results. LR, DO, EB, and 
PV conceived the study and interpreted the results. AAK, $\mathrm{MN}, \mathrm{MH}, \mathrm{FD}$ and MA designed the study and interpreted the results. SS drafted the manuscript and all other authors revised the manuscript for important intellectual content. All authors have agreed on submission to Clinical Epidemiology, reviewed and agreed on all versions of the manuscript, including the final version, and agreed to be accountable for all the contents of the article.

\section{Disclosure}

LR is an employee of Pfizer, and DO, EB, and PV are employees of Merck Healthcare KGaA, Darmstadt, Germany. FD reports receiving research grants from Pfizer, Ipsen, MSD and The Health Research Foundation of Central Denmark Region. AAK reports honoraria from AstraZeneca A/S for speaking at a Nordic-Baltic scientific meeting, "1ST Nordic/Baltic multidisciplinary scientific exchange meeting on treatment of inoperable stage III NSCLC patients" (2019). The authors report no other conflicts of interest in this work.

\section{References}

1. Escudier B, Porta C, Schmidinger M, et al. Renal cell carcinoma: ESMO Clinical Practice Guidelines for diagnosis, treatment and follow-up. Ann Oncol. 2016;27(suppl 5):v58-v68. doi:10.1093/ annonc/mdw328

2. Reck M, Popat S, Reinmuth N, et al. Metastatic non-small-cell lung cancer (NSCLC): ESMO Clinical Practice Guidelines for diagnosis, treatment and follow-up. Ann Oncol. 2014;25:iii27-39. doi:10.1093/ annonc/mdu199

3. da Silva JL, Dos Santos ALS, Nunes NCC, et al. Cancer immunotherapy: the art of targeting the tumor immune microenvironment. Cancer Chemother Pharmacol. 2019;84 (2):227-240. doi:10.1007/s00280-019-03894-3

4. Postow MA, Sidlow R, Hellmann MD. Immune-Related Adverse Events Associated with Immune Checkpoint Blockade. N Engl $J$ Med. 2018;378(2):158-168. doi:10.1056/NEJMra1703481

5. European Medicines Agency. New Treatment for Advanced Melanoma [Press Release]. European Medicines Agency; 2015.

6. Bilgin B, Sendur MAN, Hizal M, Yalcin B. An update on immunotherapy options for urothelial cancer. Expert Opin Biol Ther. 2019;19(12):1265-1274. doi:10.1080/14712598.2019.1667975

7. Kono K, Nakajima S, Mimura K. Current status of immune checkpoint inhibitors for gastric cancer. Gastric Cancer. 2020;23 (4):565-578. doi:10.1007/s10120-020-01090-4

8. Fashoyin-Aje L, Donoghue M, Chen H, et al. FDA Approval Summary: pembrolizumab for Recurrent Locally Advanced or Metastatic Gastric or Gastroesophageal Junction Adenocarcinoma Expressing PD-L1. Oncologist. 2019;24(1):103-109. doi:10.1634/ theoncologist.2018-0221

9. Tang J, Yu JX, Hubbard-Lucey VM, Neftelinov ST, Hodge JP, Lin Y. Trial watch: the clinical trial landscape for PD1/PDL1 immune checkpoint inhibitors. Nat Rev Drug Discov. 2018;17(12):854-855. doi:10.1038/nrd.2018.210

10. Rashdan S, Gerber DE. Immunotherapy for non-small cell lung cancer: from clinical trials to real-world practice. Transl Lung Cancer Res. 2019;8(3):202-207. doi:10.21037/tlcr.2018.09.15
11. Schmidt M, Schmidt SAJ, Adelborg K, et al. The Danish health care system and epidemiological research: from health care contacts to database records. Clin Epidemiol. 2019;11:563-591. doi:10.2147/ CLEP.S179083

12. Gjerstorff ML. The Danish Cancer Registry. Scand J Public Health. 2011;39(7 Suppl):42-45. doi:10.1177/1403494810393562

13. Schmidt M, Schmidt SA, Sandegaard JL, Ehrenstein V, Pedersen L, Sorensen HT. The Danish National Patient Registry: a review of content, data quality, and research potential. Clin Epidemiol. 2015;7:449-490. doi:10.2147/CLEP.S91125

14. Schmidt M, Pedersen L, Sorensen HT. The Danish Civil Registration System as a tool in epidemiology. Eur J Epidemiol. 2014;29 (8):541-549. doi:10.1007/s10654-014-9930-3

15. Mainz J, Hess MH, Johnsen SP. The Danish unique personal identifier and the Danish Civil Registration System as a tool for research and quality improvement. Int $j$ Quality Health Care. 2019;31(9):717-720.

16. Compton CC, Byrd DR, Garcia-Aguilar J, Kurtzman SH, Olawaiye A, Washington MK. AJCC Cancer Staging Atlas. New York: Springer; 2012.

17. Witjes JA, Bruins HM, Cathomas R, et al. EAU Guidelines on Muscle-Invasive and Metastatic Bladder Cancer. Arnhem, The Netherlands: EAU Guidelines Office; 2021.

18. Charlson ME, Pompei P, Ales KL, MacKenzie CR. A new method of classifying prognostic comorbidity in longitudinal studies: development and validation. J Chronic Dis. 1987;40(5):373-383. doi:10.1016/0021-9681(87)90171-8

19. Bjerregaard B, Larsen OB. The Danish Pathology Register. Scand J Public Health. 2011;39(7 Suppl):72-74. doi:10.1177/1403494810393563

20. Erichsen R, Lash TL, Hamilton-Dutoit SJ, Bjerregaard B, Vyberg M, Pedersen L. Existing data sources for clinical epidemiology: the Danish National Pathology Registry and Data Bank. Clin Epidemiol. 2010;2:51-56. doi:10.2147/CLEP.S9908

21. Kaplan EL, Meier P. Nonparametric Estimation from Incomplete Observations. J Am Stat Assoc. 1958;53(282):457-481. doi:10.1080/ 01621459.1958.10501452

22. Broe MO, Jensen PB, Mattsson TO, Pottegard A. Validity of Antineoplastic Procedure Codes in the Danish National Patient Registry: the Case of Colorectal Cancer. Epidemiology. 2020;31 (4):599-603. doi:10.1097/EDE.0000000000001208

23. Lund JL, Froslev T, Deleuran T, et al. Validity of the Danish National Registry of Patients for chemotherapy reporting among colorectal cancer patients is high. Clin Epidemiol. 2013;5:327-334. doi:10. 2147/CLEP.S49773

24. Pedersen SA, Schmidt SAJ, Klausen S, et al. Melanoma of the Skin in the Danish Cancer Registry and the Danish Melanoma Database: a Validation Study. Epidemiology. 2018;29(3):442-447. doi:10.1097/ EDE.0000000000000802

25. Deleuran T, Sogaard M, Froslev T, et al. Completeness of TNM staging of small-cell and non-small-cell lung cancer in the Danish cancer registry, 2004-2009. Clin Epidemiol. 2012;4(Suppl 2):39-44. doi:10.2147/CLEP.S33315

26. de Alencar Camara Vieira FM, Victorino A. Treatment Patterns Among Patients with Metastatic and/or Unresectable Gastric Cancer in Brazil. J Gastrointest Cancer. 2019;50(4):780-793. doi:10.1007/ s12029-018-0145-9

27. Jessmon P, Boulanger T, Zhou W, Patwardhan P. Epidemiology and treatment patterns of epithelial ovarian cancer. Expert Rev Anticancer Ther. 2017;17(5):427-437. doi:10.1080/14737140.2017.1299575

28. Pal S, Gong J, Mhatre SK, et al. Real-world treatment patterns and adverse events in metastatic renal cell carcinoma from a large US claims database. BMC Cancer. 2019;19(1):548. doi:10.1186/s12885019-5716-z

29. Harada K, Nozawa M, Uemura M, et al. Treatment patterns and outcomes in patients with unresectable or metastatic renal cell carcinoma in Japan. Int J Urol. 2019;26(2):202-210. doi:10.1111/iju. 13830 
30. Wagstaff J, Jones R, Hawkins R, et al. Treatment patterns and clinical outcomes in patients with renal cell carcinoma in the UK: insights from the RECCORD registry. Ann Oncol. 2016;27(1):159-165. doi:10.1093/annonc/mdv504

31. Simeone JC, Nordstrom BL, Patel K, Klein AB. Treatment patterns and overall survival in metastatic non-small-cell lung cancer in a real-world, US setting. Future Oncol. 2019;15(30):3491-3502. doi:10.2217/fon-2019-0348

32. Om KRIS - Koordineringsrådet for ibrugtagning af sygehusmedicin. Available form: https://www.regioner.dk/kris/om-kris. Accessed September 21, 2020.

33. Om os. Medicinraadet.DK. Available form: https://medicinraadet.dk/ om-os. Accessed September 21, 2020.

34. Richters A, Mehra N, Meijer RP, et al. Utilization of systemic treatment for metastatic bladder cancer in everyday practice: results of a nation-wide population-based cohort study. Cancer Treat Res Commun. 2020;25:100266. doi:10.1016/j.ctarc.2020.100266
35. Ward MM, Ullrich F, Matthews $\mathrm{K}$, et al. Who does not receive treatment for cancer? J Oncol Pract. 2013;9(1):20-26. doi:10.1200/ JOP.2012.000829

36. Jiang Y, Chen M, Nie H, Yuan Y. PD-1 and PD-L1 in cancer immunotherapy: clinical implications and future considerations. Hum Vaccin Immunother. 2019;15(5):1111-1122. doi:10.1080/ 21645515.2019.1571892

37. Jumeau R, Vilotte F, Durham AD, Ozsahin EM. Current landscape of palliative radiotherapy for non-small-cell lung cancer. Transl Lung Cancer Res. 2019;8(Suppl 2):S192-S201. doi:10.21037/tlcr.2019. 08.10
Clinical Epidemiology

\section{Publish your work in this journal}

Clinical Epidemiology is an international, peer-reviewed, open access, online journal focusing on disease and drug epidemiology, identification of risk factors and screening procedures to develop optimal preventative initiatives and programs. Specific topics include: diagnosis, prognosis, treatment, screening, prevention, risk factor modification,

Submit your manuscript here: https://www.dovepress.com/clinical-epidemiology-journal

\section{Dovepress}

systematic reviews, risk \& safety of medical interventions, epidemiology \& biostatistical methods, and evaluation of guidelines, translational medicine, health policies \& economic evaluations. The manuscript management system is completely online and includes a very quick and fair peer-review system, which is all easy to use. 